\title{
(Un-)besetzte Räume
}

Rezension zu Friederike Landau / Lucas Pohl / Nikolai Roskamm (Hg.) (2021):

[Un]Grounding. Post-foundational geographies. Bielefeld: transcript.

Abb. 1 Titel des Buches (Quelle: transcript-Verlag)

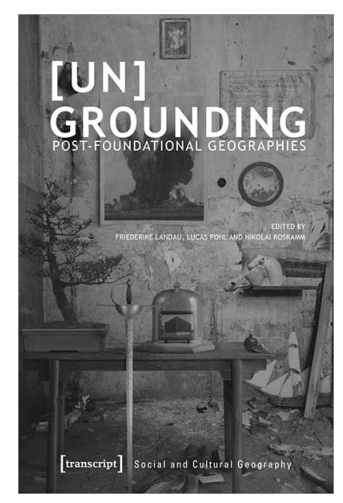

Die Herausgeber_innen von[Un]Grounding.Post-foundationalgeographies eröffnen ihr Buch mit der Losung postfundamentalistischen Denkens: Es gibt keinen letzten Grund des Sozialen: Kein Gott, keine biologischen Gesetze, kein genetischer Code, kein Markt, keine anthropologische Essenz, kein Produktionsverhältnis bildet den letzten Grund für soziale und historische Akteur_innen (Landau/Pohl/Roskamm 2021b: 9). Jene Gründungsfigur - die stets anwesende Abwesenheit - bildet den Ausgangspunkt für die 15 englischsprachigen Beiträge des Sammelbands, der in drei Abschnitte unterteilt ist: (1) „Theoretical (Re)Positionings“, (2) „,[Un]Grounding Geographies“ und (3) „Post-Foundationalism in the City“.

Obgleich die Negativität konstitutiv für jede Form sozialer Gründung ist, bedeutet dies nicht, dass postfundamentalistisches Denken jegliche Formen des Gründens ablehnt. Wie auch dieser Band zeigt, geht es im postfundamentalistischen Denken um eine Suche nach den Bedingungen der (Un-)Möglichkeit des Gründens selbst. Mit Gründung ist dabei die stets aufs Neue anstehende Institution gesellschaftlicher Verhältnisse gemeint, deren Bewegung nie ganz zum Stillstand kommt. Bemerkenswert ist aus Sicht der am postfundamentalistischen Denken interessierten Leserin, dass diese (Un-)Möglichkeit in dem rezensierten Band durchgehend innerhalb der Dimensionen des Räumlichen verortet wird. Obwohl es durchaus üblich ist, postfundamentalistische Denker_innen wie Ernesto Laclau und Chantal Mouffe, Alain Badiou, Jacques Derrida, Claude Lefort oder Jacques Rancière (vgl. Landau/Pohl/Roskamm 2021b: 9) im Bereich der Politischen Theorie, der Gesellschaftstheorie oder der Sozialtheorie zu verorten, ist es weniger üblich, ihre Theorien explizit als Raumtheorien zu begreifen. Dies bedeutet allerdings nicht, dass die Verbindung zwischen Raum und postfundamentalistischem Denken neu wäre. Der Sammelband bietet Anknüpfungspunkte an bereits geführte Debatten, wie etwa die zwischen Ernesto Laclau und Doreen Massey über Raum, Diskurs und Hegemonie. In diese Kerbe schlägt auch dieses Buch, in dem es nicht nur zeigt, dass der Raum eine zentrale Rolle in postfundamentalistischem Denken spielt, sondern, dass eine Auseinandersetzung zwischen Politischer Theorie, human geography 
und urban studies äußerst fruchtbar zu sein scheint. Ziel der Herausgeber_innen ist es, räumliche Politiken, politische Räume und Politiken des Raumes (Landau/Pohl/Roskamm 2021b: 10) miteinander zu verbinden sowie Möglichkeiten eines postfundamentalistischen räumlichen Denkens auszuloten. Postfundamentalismus begegnet dem spatial turn.

Im ersten Abschnitt „Theoretical (Re)Positionings” werden räumliche Dimensionen in den Theorien von Jacques Lacan, Henri Lefebvre, Ernesto Laclau, Alain Badiou und Jacques Rancière herausgearbeitet. Für die Kenner_innen postfundamentalistischer Texte ergibt sich hier womöglich ein Aha-Moment, - denn Begriffe wie „Dislokation“ (Laclau), „Bühne“ (Rancière) oder „Inklusion/Exklusion“ (Mouffe) können räumlich verortet und verstanden werden, obgleich es jedoch eher ungewöhnlich ist, dass sie von politischen Theoretiker_innen als räumlich interpretiert werden. Mark Davidson und Kurt Iveson (2021) beispielsweise weisen in ihrem Beitrag „Spacing Rancière's Politics“ darauf hin, dass Rancières Politikverständnis und die damit einhergehenden Inklusions- und Exklusionsprozesse auch territorial verstanden werden können (ebd.). Die von Rancière postulierte Zählung und Verrechnung in politischen Artikulationsprozessen berge auch ein Potenzial, soziale Geographien neu zu definieren (ebd.: 135). Lucas Pohl und Erik Swyngedouw (2021) verweisen in ihrem Beitrag „The World and the Real: Space and the Political after Lacan" auf die (theoriegeschichtliche) Bedeutung von Jacques Lacans Register des Realen für postfundamentalistisches Denken. Dabei wird jedoch nicht ganz deutlich, welche Rolle das Räumliche in Hinblick auf die Lacan'schen Dimensionen des Symbolischen, Imaginären und Realen spielt. Der Begriff des Raumes taucht im Text immer wieder auf und wird dann wieder umkreist. Mit Absicht?

In seinem Beitrag „Institution and Dislocation: Philosophical Roots of Laclau's Discourse Theory of Space and Antagonism“ formuliert Oliver Marchart (2021) eine Neuinterpretation von Laclaus Raumverständnis. Anders als Doreen Massey, die Laclau ein passives Konzept von Raum zuschreibt (Massey 1992: 66 f.), versucht Marchart die dislozierenden Dimensionen von Raum aufzuzeigen. Um die Opposition zwischen Raum (passiv) und Zeit (aktiv) zu dekonstruieren, folgt Marchart den radikalen Implikationen von Laclaus Antagonismus-Begriff und entwickelt ein Verständnis von Zeit und Raum als zwei verschiedener Modi radikaler Negativität. Lesenswert und in gewisser Weise originell ist auch der Beitrag von Jens Kaae Fisker (2021), der die feministische Wirtschaftsgeographin J. K. Gibson-Graham als postfundamentalistische Denkerin vorstellt. Raum wird hier als schwangere Negativität beschrieben. Der Text wirft die Frage auf, warum so wenig Auseinandersetzung zwischen queer-feministischer Theorie und postfundamentalistischem Denken trotz geteilter Grundsätze stattfindet. Obgleich Fisker sich unter Verweis auf andere feministische Autor_innen eine stärkere Bezugnahme zwischen feministischen und postfundamentalistischen Perspektiven wünscht, gibt er nur wenig Hinweise darauf, warum eine Verbindung sinnvoll oder fruchtbar wäre. Das ist schade, nicht zuletzt, da die Herausgeber_innen schon in ihrer Einleitung darauf hinweisen, dass Postfundamentalismus immer schon die Tendenz aufwies, ein „men's club“ (Landau/Pohl/Roskamm 2021b: 30) zu sein, ohne möglichen Gründen hierfür nachzugehen. Dieses Problem spiegelt 
sich auch in der Zusammenstellung des Sammelbandes wider: Nur drei der 15 Beiträge wurden von Frauen (mit-)verfasst. In der Einleitung geben die Herausgeber_innen an, dass es wichtiger werden würde, die Grenzen postfundamentalistischer Theorien zu schubsen und zu drücken (,push and squeeze"), bis feministische und post-koloniale Zugänge sowie Positionen von Black, Indigenous and People of Colour (BIPOC) sowie African, Latino/ Hispanic, Asian, Native American (ALANA) stärker einbezogen werden (Landau/Pohl/Roskamm 2021b: 30).

Aus der feministischen Perspektive der Rezensentin wäre beispielsweise der stärkere Einbezug von Frauen in die Autor_innenschaft der Beiträge eine Möglichkeit, die Grenzen postfundamentalistischen Denkens auszuweiten. Konkrete Bemühungen, den Erfahrungen und theoretischen Zugängen von Frauen, Migrant_innen und Arbeiter_innen in der Gegenwart räumlicher Verortung mehr Raum zu geben, würde womöglich einen Reflexionsprozess anstoßen, der die theoretischen Konzepte selbst verändern oder auf die Probe stellen könnte.

Hier zeigt sich eine gewisse Scheu des Postfundamentalismus vor bestimmten Allianzen, die womöglich in der Annahme begründet ist, dass der Kern postfundamentalistischen Denkens - die Abwesenheit einer letzten Gründung - durch starke (zum Beispiel feministische oder antirassistische) Gründungsnarrative schnell in den Hintergrund rücken könnte. Obwohl das postfundamentalistische Denken offen für Gründungen ist, erscheint es sein wesentliches Anliegen zu sein, unentwegt auf die (ent-)gründende Negativität zu verweisen, die nicht auf Fragen der Legitimierung und/oder Kritik der Verteilung und Zuordnung von Rollen und Räume reduziert werden kann. Es tut sich hier ein grundlegender Konflikt postfundamentalistischen Denkens auf. Der Postfundamentalismus verweist auf das, was sich der Politik entzieht. Zugleich will er jedoch kein Philosophismus sein, der vorgibt, politische Auseinandersetzungen von einem Elfenbeinturm aus zu betrachten. Genauso wenig will er ein Politizismus sein, der sich einer bestimmten politischen Positionierung verschreibt oder Theorie ausschließlich mit Blick auf ihre Brauchbarkeit oder gar Verwertbarkeit für politische Ziele beurteilt. Wie soll der Postfundamentalismus mit dieser Spannung umgehen? Seine Stärke liegt mit Sicherheit nicht in einer Auflösung dieser Spannung, sondern vielmehr im produktiven Umgang mit derselben. Da sich diese Spannung auch durch die Beiträge des Sammelbandes zieht, werden diejenigen Leser_innen enttäuscht sein, die konkrete linke Positionierungen erwarten. Der Band bietet keine konkreten Anweisungen für Strategien linker Raumpolitik und stellt auch keine wissenschaftliche Auseinandersetzung mit Policy-Fragen rund um räumliche Gestaltung dar. Vielmehr verweisen die Texte auf Möglichkeiten, (Neu-)gründungen des Sozialen im Hier (räumlich) und Jetzt (zeitlich) zu verankern. Das emanzipatorische Potenzial liegt in den Verweisen darauf, dass politische Handlungsspielräume nie ganz geschlossen sind.

Im zweiten Abschnitt „,[Un]Grounding Geographies“ wird postfundamentalistische Raumtheorie durch die Verbindung mit politisch-theoretischen Konzepten um das Vokabular geographischen Wissens erweitert. Beispielsweise fragt Frederike Landau (2021) in ihrem Beitrag „,[Un]Grounding Agonistic Public Space: Approaching Mouffe's Spatial Theory via Museums“ nach den veräumlichten Dimensionen von Agonistik. Dazu untersucht sie 
die Rolle radikaler Museen für die Herstellung kritischer urbaner Infrastruktur anhand eines Museums in Indien. Mit dem Beitrag „Always Geographize! Frederic Jameson and Political Space“ gibt Clint Burnham (2021) dem Politisch-Unbewussten eine räumliche Dimension. Er fragt nach Möglichkeiten, das geographische Unbewusste zu dekolonialisieren. Das ist nicht zuletzt deswegen interessant, weil postfundamentalistisches Denken theoriegeschichtlich eher durch eine Verschiebung von der Geschichte bzw. Ökonomie zur Ontologie gekennzeichnet ist (Stichwort „Always Ontologize!"). Burnhams Beitrag wirft also die Frage auf: Was leisten die postfundamentalistischen Verschiebungen von der Geschichte zur Ontologie, zur Geographie? Wie unterscheiden sich Perspektiven, Ideen oder Zugänge je nach gewählter Linse? Dieser Beitrag ist trotz einiger voraussetzungsvoller Textpassagen und abstrakter Formulierungen nicht zuletzt aufgrund seiner Aktualität lesenswert. Burnham verweist unter anderem auf residential schools in Kanada, Internatsschulen für Kinder der indigenen Bevölkerung Kanadas, die ab der zweiten Hälfte des 19. Jahrhunderts bis in die 1990er Jahre betrieben wurden. Kurz nach der Veröffentlichung dieses Sammelbands im Mai 2021 gerieten die residential schools (Burnham spricht vom „räumlichen Unbewussten Kanadas") ins Licht medialer Aufmerksamkeit - nachdem auf einem Schulgelände mehr als 200 Kinderleichen in Massengräbern gefunden worden waren.

Im letzten Abschnitt „Post-Foundationalism in the City“ wird postfundamentalistisches Denken mit umkämpftem Raum in der Stadt und Stadtpolitiken verknüpft. Diese Verbindung ist mit Sicherheit nicht neuartig. Auch dieser Teil des Buches zeigt, dass die Verknüpfung von postfundamentalistischer Theorie mit radikaler Demokratietheorie und Stadtpolitiken naheliegend ist und funktionieren kann. Davon zeugt auch der Text „(Non) Building Alliances: Approaching Urban Politics through Siegfried Kracauer's Concept of Nonsolution“ von Gabu Heindl und Drehli Robniks (2021). Er eröffnet einen Zugang zu Stadtpolitik, der sich auf die Paradoxien politischer Handlungsfähigkeit einlässt. Dabei beziehen sich die Autor_innen auf das Konzept der „Nicht-Lösung“ des Schriftstellers und Architekten Siegfried Kracauer und heben damit hervor, dass es keine endgültige (architektonische) Lösung für ein egalitäreres Zusammenleben gibt, da jede Lösung auch gleichzeitig ein „Nicht“ enthält (Heindl/Robnik 2021: 251). Die Autor_innen zeigen jedoch auch, dass diese Unmöglichkeit einer wirklich für alle befriedigenden Lösung gleichzeitig die Möglichkeit birgt, immer wieder neu anzufangen und emanzipatorische Politik voranzutreiben. Heindl und Robnik zeigen mit Verweis auf einige architektonische Initiativen in Wien, die in Allianz mit sozialen Bewegungen entstanden sind, dass „mourning as utopian testimonial“ (ebd.: 255) mehr sein kann, als bloßer Katzenjammer.

Die Forschungsnetzwerke der Herausgeber_innen Friederike Landau, Lucas Pohl und Nikolai Roskamm liegen im Bereich der Raumtheorie, der Politischen Theorie, der Geographie sowie der Stadtforschung. Nikolai Roskamm (2017) hatte zuletzt einen wichtigen Beitrag zur Debatte mitseinem Buch Die unbesetzte Stadt vorgelegt. Der Austausch zwischen unterschiedlichen Forschungsbereichen spiegelt sich auch in der Auswahl der Beiträge wider. Am Verfassen der Beiträge waren Angehörige unterschiedlicher Statusgruppen beteiligt; der Band enthält Beiträge etablierterer und weniger etablierterer 
Forscher_innen und trägt damit zu einem Abbau von Hierarchien in der akademischen Zusammenarbeit bei. Der Sammelband ist an einer interdisziplinären Schnittstelle angesiedelt und lesenswert für all jene Leser_innen, die an politischer (postfundamentalistischer) Theorie interessiert sind oder sich für Geographie und/oder urban studies interessieren.

\section{Autor_innen}

Valerie Scheibenpflug ist politische Theoretikerin. Sie forscht zu politischer Philosophie, Theorien des Demos und Grenzregimen.

valerie.scheibenpflug@univie.ac.at

\section{Literatur}

Burnham, Clint (2021): Always geographize! Frederic Jameson and political space. In: Friederike Landau / Lucas Pohl / Nikolai Roskamm (Hg.), [Un]Grounding. Post-foundational geographies. Bielefeld: transcript, 175-196.

Davidson, Mark / Iveson, Kurt (2021): Spacing Rancière's politics. In: Friederike Landau / Lucas Pohl / Nikolai Roskamm (Hg.), [Un]Grounding. Post-foundational geographies. Bielefeld: transcript, 133-150.

Fisker, Jens Kaae (2021): Encountering post-foundationalism in J. K. Gibson-Graham's space of pregnant negativity or, ungrounding the ground itself. In: Friederike Landau / Lucas Pohl / Nikolai Roskamm (Hg.), [Un]Grounding. Post-foundational geographies. Bielefeld: transcript, 63-80.

Heindl, Gabu / Robnik, Drehli (2021): (Non)building alliances: Approaching urban politics through Siegfried Kracauer's concept of nonsolution. In: Friederike Landau / Lucas Pohl / Nikolai Roskamm (Hg.), [Un]Grounding. Post-foundational geographies. Bielefeld: transcript, 243-260.

Landau, Friederike (2021): [Un]Grounding agonistic public space: Approaching Mouffe's spatial theory via museums. In: Friederike Landau / Lucas Pohl / Nikolai Roskamm (Hg.), [Un]Grounding. Post-foundational geographies. Bielefeld: transcript, 153-174.

Landau, Friederike/ Pohl, Lucas /Roskamm, Nikolai (Hg.) (2021a): [Un]Grounding. Post-foundational geographies. Bielefeld: transcript.

Landau, Friederike / Pohl, Lucas / Roskamm, Nikolai (2021b): Introduction. In: Friederike Landau / Lucas Pohl / Nikolai Roskamm (Hg.), [Un]Grounding. Post-foundational geographies. Bielefeld: transcript, 9-40.

Marchart, Oliver (2021): Institution and dislocation: Philosophical roots of Laclau's discourse theory of space and antagonism. In: Friederike Landau / Lucas Pohl / Nikolai Roskamm (Hg.), [Un]Grounding. Post-foundational geographies. Bielefeld: transcript, 99-116.

Massey, Doreen (1992): Politics and space/time. New Left Review I/196, 65-84.

Pohl, Lucas / Swyngedouw, Erik (2021): The world and the real: Space and the political after Lacan. In: Friederike Landau / Lucas Pohl / Nikolai Roskamm (Hg.), [Un]Grounding. Post-foundational geographies. Bielefeld: transcript, 43-62.

Roskamm, Nikolai (2017): Die unbesetzte Stadt. Postfundamentalistisches Denken und das urbanistische Feld. Basel: Birkhäuser. 